\title{
LA-UR-20-20487
}

Approved for public release; distribution is unlimited.

Title: $\quad$ UC LANL Postdoc Entrepreneur Fellowship Cohort Three

Author(s): $\quad$ Cernicek, Mary Beth

Intended for: $\quad$ Report

Issued: 
Disclaimer:

Los Alamos National Laboratory, an affirmative action/equal opportunity employer, is operated by Triad National Security, LLC for the National Nuclear Security Administration of U.S. Department of Energy under contract 89233218CNA000001. By approving this article, the publisher recognizes that the U.S. Government retains nonexclusive, royalty-free license to publish or reproduce the published form of this contribution, or to allow others to do so, for U.S. Government purposes. Los Alamos National Laboratory requests that the publisher identify this article as work performed under the auspices of the U.S. Department of Energy. Los Alamos National Laboratory strongly supports academic freedom and a researcher's right to publish; as an institution, however, the Laboratory does not endorse the viewpoint of a publication or guarantee its technical correctness. 


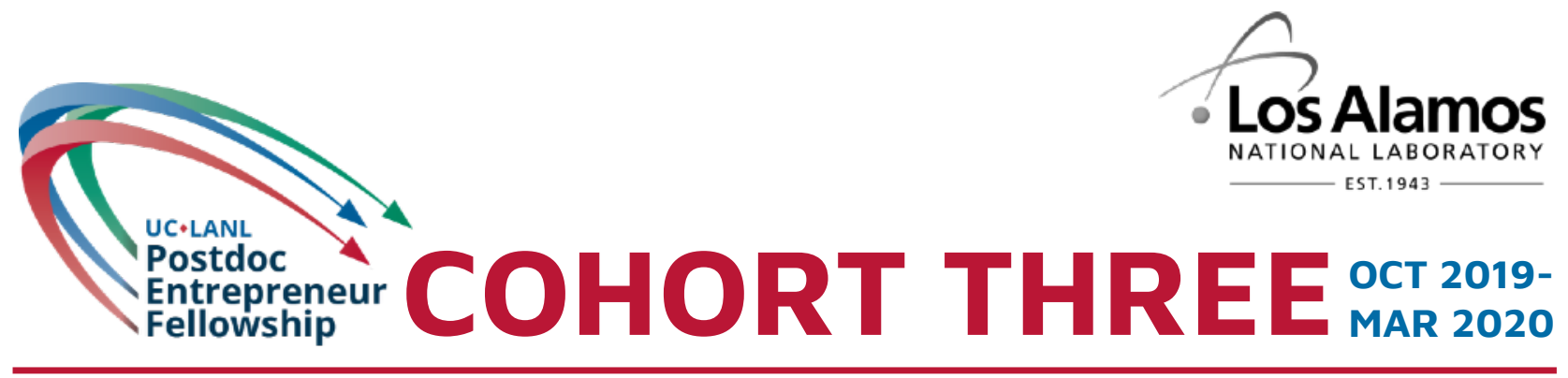

Three Laboratory postdoctoral researchers have been selected for this year's cohort of Entrepreneur Fellows. The six-month fellowship program - a joint initiative of the University of California and Los Alamos, now in its third year - supports early career scientists explore applications and evaluate market opportunities for their technologies. The Lab selects Entrepreneur Fellows based on the potential for their projects to have both scientific and commercial impact. The Fellows receive six months of facilitated training and mentoring, as well as funding to engage in customer discovery, market exploration and technology advancement.

"It has been thrilling to witness the growing interest in the UC/Los Alamos Entrepreneurial

Postdoctoral Fellowship program. Year over year, the postdoctoral fellows selected to participate in this program have been exceptional. We look forward to seeing these fellows playing a leading role in fostering innovation and research within the Los Alamos National Laboratory, as well as to extend and amplify the progress for creating a thriving tech transfer ecosystem in the Northern New Mexico region and beyond."

June Yu, Ph.D.

Executive Director, National Laboratory Programs University of California's Office of the President

\section{9-20 COHORT}

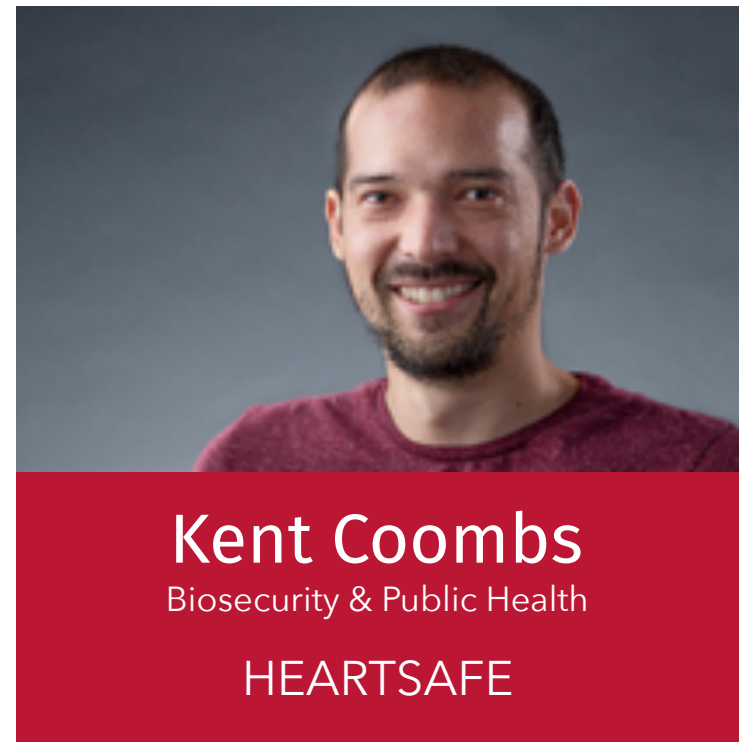

HeartSafe, a revolutionary new tool for testing novel drug toxicity and efficacy, is an advanced artificial heart system that accurately predicts the effects of drugs on the human heart. This system is better and more affordable than animal testing and has the potential to revolutionize the drug development world by replacing animal models entirely. Los Alamos' unique engineering and biological capabilities when combined with human cell samples, have resulted in a model system that is superior to current "organ on a chip" models and offers a new testing method that will give critical information earlier in the drug development pipeline. 


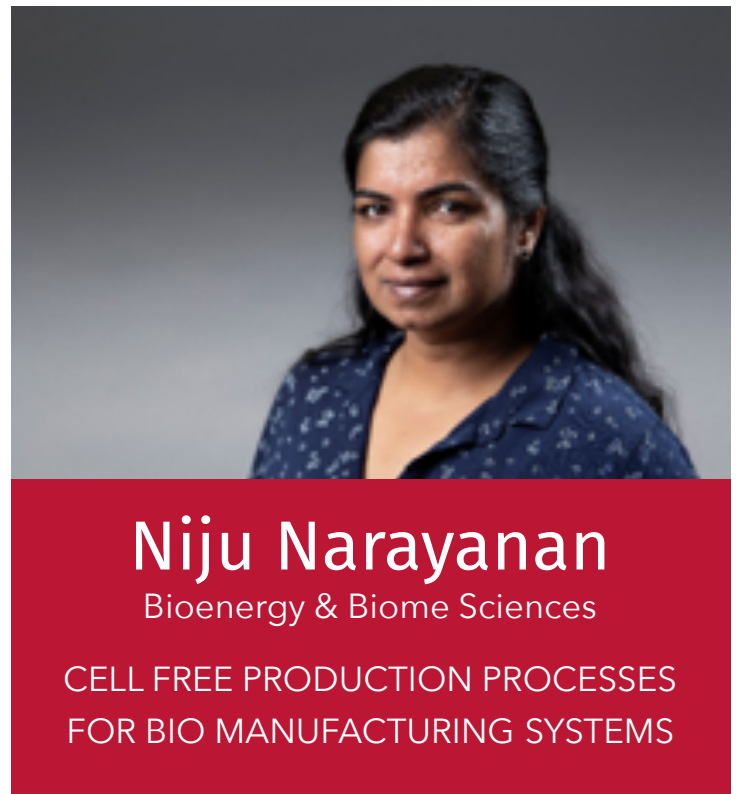

Bio-based manufacturing systems experience high costs and low efficiencies due to living cells desire for optimal and continued growth that is in direct conflict with engineered processes to produce the best titers in target molecules. We are designing a screening platform coupled to our biosensor to customize production processes in a cell free environment that offers manufactures higher titers and yields, better resource utilization and controlled reactions. We are targeting the optimization of high value molecules in food replacements such as pigments, scents, therapeutics, flavors and fragrances.

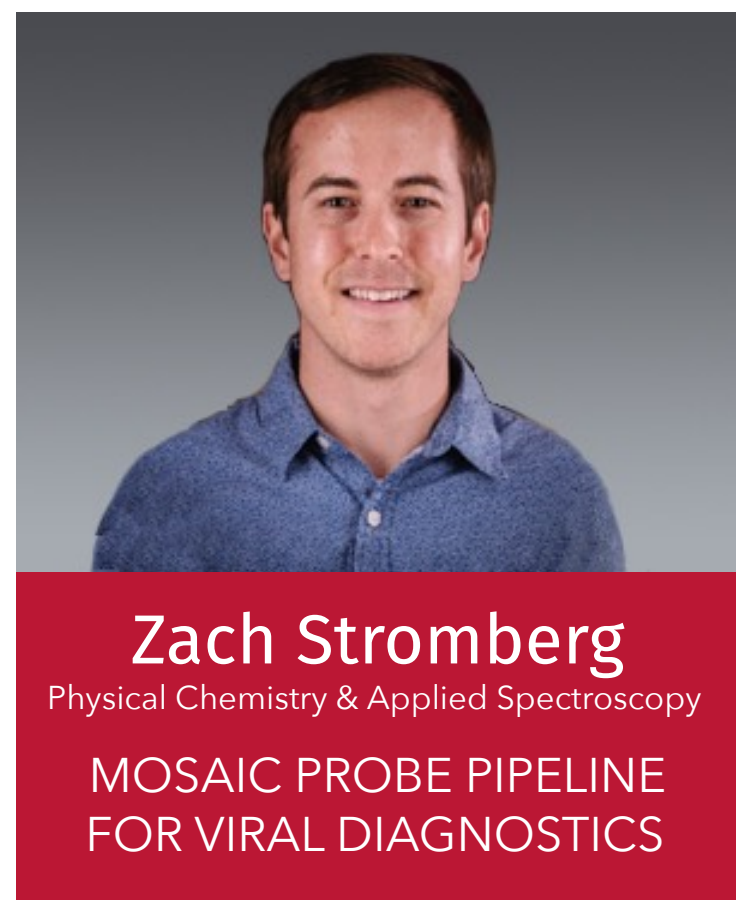

The viral mosaic probe pipeline has been developed to create high-coverage probes for detection of emerging viruses. Current commercial probes for viral diseases can be insensitive because of high genetic diversity, which results in false negative results and a missed diagnosis. In addition, there is increased demand for probes that can function in highly multiplexed settings. Our technology is based on the theoretical mosaic design (used in HIV mosaic vaccine design) and has been reconfigured to create high-coverage multiplexed probes that target viruses. Probes are compatible with existing diagnostic instruments (e.g., PCR) and can be used in our LANL biosensor (the basis for the Universal Bacterial Sensor). Overall, our platform generates molecular probes that target highly diverse and rapidly mutagenic viruses and can be used for highly sensitive detection that can be easily expanded to other pathogens for both diagnostics and surveillance. 